\title{
Starting Point of Cluster-Derived Silicon Nanowires
}

\author{
Aijiang Lu \\ Science College, Donghua University, Shanghai, China \\ Email: ajlu@dhu.edu.cn
}

Received January 13, 2013; revised February 12, 2013; accepted February 21, 2013

Copyright (c) 2013 Aijiang Lu. This is an open access article distributed under the Creative Commons Attribution License, which permits unrestricted use, distribution, and reproduction in any medium, provided the original work is properly cited.

\begin{abstract}
The assembly of medium-sized silicon nanoclusters was simulated to study the starting point of the formation of clusterderived silicon nanowires (CDSiNWs). Hydrogen-terminated clusters were found repulsing each other and inter-connecting through the hydrogen bonds, thus could not form a stable silicon nanowire (SiNW). Between the pristine silicon clusters without hydrogen saturation, the assembly takes place automatically. An orientation priority in cluster assembly is obtained, as silicon clusters Si29 are more possibly adhered along $<111>$ direction than the other directions. Such an assembly may be the starting point of the SiNW growth along $<111>$ direction. Moreover, it indicates the possibility of silicon tetrapods or zigzag wires formation, besides straight SiNWs.
\end{abstract}

Keywords: Cluster; Nanowire; First Principle Calculation

\section{Introduction}

Silicon nanowire (SiNW) is not a new topic as it has been studied for decades due to the potential applications in electric, electronic, optoelectronic and sensing fields [1-6]. But the most basic problem, the wire growth has not been demonstrated clearly. Much effort has been taken as a number of reports on synthesis or characteristic of SiNWs are available [7,8]. As reported, SiNWs along $<110\rangle,<111>$ and $<112>$ directions were successfully synthesized in experiments and the diameters of them range between 1.3 to $7 \mathrm{~nm}$. The quasi-1D material was regarded as a good candidate to show the quantum confinement in real systems $[9,10]$. However, the growth direction of the SiNWs exhibits complexity, according to the different methods and conditions in the synthesis [11, 12]. For example, in Vapor-Liquid-Solid (VLS) method the small sized SiNWs (diameter less than $10 \mathrm{~nm}$ ) prefer $<110>$ [13] direction, and the NWs with diameter larger than $20 \mathrm{~nm}$ prefer the growth direction of $<111>$ [14]. In the etching process of (110) silicon substrate the crystallographically of vertical wires prefers $<100\rangle$ direction [15]. By thermal evaporation, non-unique crystallographic is found by Ma et al. [16]. The bond orientational order was reported to influence the physical properties of SiNWs heavily $[17,18]$, so the direction of SiNWs need to be determined first before they were used in real applications.

Thus, the motivation and procedure of the SiNWs growth has been interested in widely. Although the oxygen assisting, etching procedure and metallic catalyst were suggested to explain the growth dynamics, there has not a satisfactory answer been reached yet. Characterization of SiNWs indicates the relation of NWs with the bulk crystal. Consequently the model of SiNWs has been suggested as the cylinder structure cut from bulk silicon crystal. On the other hand, as the diameter of small sized SiNW is close to that of the medium-sized silicon cluster, cluster-derived SiNW (CDSiNW) is also concerned of as a possible structural model to study the SiNW in theory. The latter has been supported as the assembly of silicon clusters on silicon surfaces confirmed with Scanning Tunneling Microscopy (STM) in experiments $[19,20]$.

The possibility of silicon clusters congregating and forming a wire-like structure was focused in this work. Si29 cluster with crystal structure was selected as the building block. Different connections between several Si29 clusters were studied. The energy analysis showed that the assembly between silicon clusters with hydrogen termination would not take place automatically. However, between the pristine silicon clusters, the connection between them results in the decrease of total energy and the bonding along $<111>$ orientation showed the lowest formation energy. The bonded structure could be the starting point of SiNWs along $<111>$ direction, instead of the model cut from bulk crystal. Moreover, our work indicated the formation of a tetrapod silicon structure or zigzag wire, besides straight SiNWs in the product of as- 
sembly.

\section{Method}

Our calculation was performed in density functional theory, based on the iterative diagonalization and plane wave basis sets. The GGA-LYP and GGA-PW91 exchangecorrelation functionals were adopted and the double numerical basis with polarization (DNP) was selected to describe the silicon atom. Norm-conserving potential was used to describe the interaction between atoms. Spin of electron was not included in this work as it was not of importance to the cluster assembly. All of the atomic structures had been full-relaxed and the interforce was less than $0.04 \mathrm{eV} / \mathrm{A}$ in the geometry optimization.

Distance between silicon clusters and the arranged orientation were controlled manually in the simulation, and no constraint was applied. Si-Si bond length in the final structure was larger than that in bulk crystal (2.3 A), which agreed with the results in some other works on silicon surface reconstruction [21].

\section{Results and Discussion}

Si29 clusters were selected as the building block with a sphere-like profile, which was built based on silicon bulk crystal. The cluster was full-relaxed and the total energy was used to calculate the formation energy of CDSiNW in this work. Hydrogen atoms were bonded on the surface to saturate the dangling bonds and the Si29H24 was used to study the $\mathrm{H}$-terminated cluster.

The assembly of $\mathrm{H}$-terminated silicon clusters were calculated first, and the initial distance between two clusters ranged from 3.0 to $0.5 \mathrm{~A}$. The approaching of the clusters along different directions has been considered, and the shortest distance between two atoms in neighboring clusters was found 1.5 A or so based on the energy analysis. Such a distance was much larger than the bond length of any chemical bonds formed by hydrogen. Thus the interaction between $\mathrm{H}$-terminated silicon clusters could be explained as van de Waals interaction. This weak connection between clusters ruled out the possibility of CDSiNWs formation with H-terminated clusters.

The silicon clusters without hydrogen saturation were studied next. The surface reconstruction was remarkable in the optimized structure of Si29, as indicated in Figure 1. The profile of the optimized cluster was close to a tetrahedron, as the (111) facets were flatter than that before the relaxation. Si-Si bond length was increased and the silicon atoms were 3 -fold or 4 -fold in the cluster.

The assembly of silicon clusters was concerned of between two clusters. The distance between them and the orientation of the arrangement were modified manually. The full relaxation was applied and no constraints were added in the systems. Two silicon clusters were arranged along different orientations and the binding energy $\mathrm{E}_{\text {tot }}{ }^{-}$ $2 * E_{\text {si29 }}$ were calculated and compared in Figure 2. In this figure, the atomic structure of the connected clusters was shown as inset just above or under the data points, and the labels (a) to (e) corresponded with the different connecting directions. Namely, they were (a) (100)-(100) dimer, (b) (100)-(110) dimer, (c) (100)-(111) dimer, (d) (110)-(110) dimer and (e) (111)-(111) dimer respectively. Here the Miller index showed the crystal surface the lateral facet lied in. This series of models were prepared through rotating one cluster around the other and laying them together. The chemical bonds between them were formed after a geometry optimization so that the cluster dimer was obtained with an inter distance in the cluster dimer.

Due to the definition of binding energy in this work, negative binding energy indicated exothermal procedure and the process often took place automatically. As displayed in Figure 2, all of the Si29-Si29 systems concerned showed negative binding energies. It was evidence that the Si29 cluster would assemble to form a larger

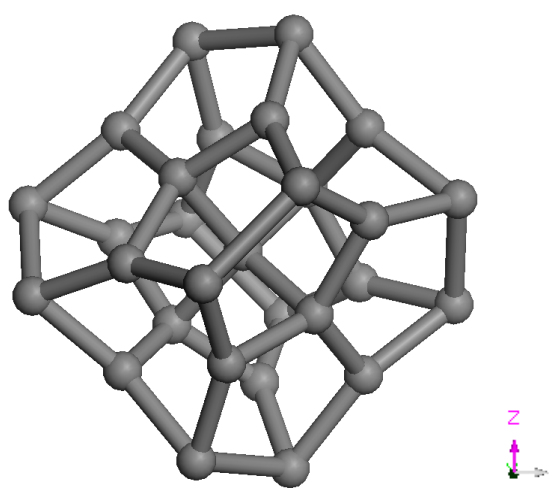

Figure 1. Atomic structure of relaxed Si29 cluster.

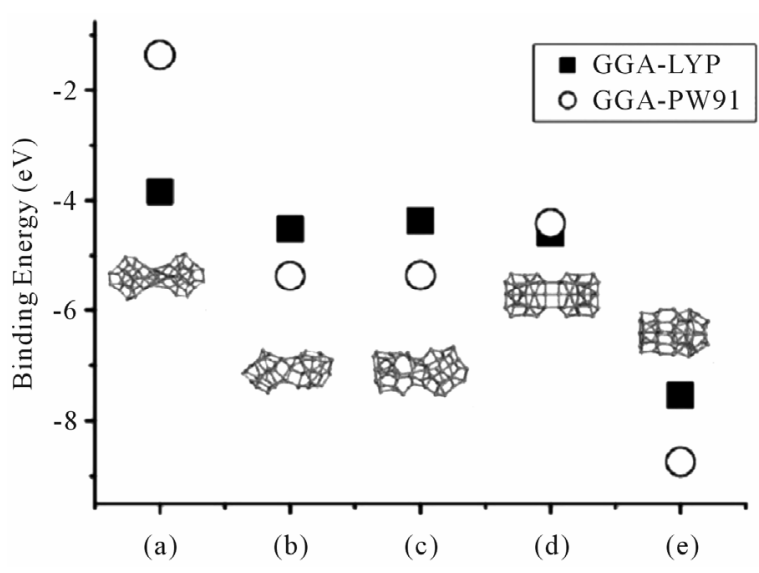

Figure 2. Binding energy of Si29-Si29 dimer. Atomic structure is shown as inset, namely (a) (100)-(100) dimer; (b) (100)-(110) dimer; (c) (100)-(111) dimer; (d) (110)-(110) dimer and (e) (111)-(111) dimer. 
cluster automatically, no matter in which direction.

However, among the cluster dimers, (111)-(111) dimer showed the lowest binding energy, which indicated that the connection of Si29 clusters in $<111>$ direction would lead to the largest decrease of the total energy. Such a result also showed that the CDSiNW would be obtained in $<111>$ direction more possibly than in other orientations, when the assembly could take place in a steady stream or a template. It was reported that the small-sized SiNWs with crystalline structure prefer $<110>$ direction in VLS method. Only when the size of the SiNW increased the SiNWs prefer to grow along $<111>$ direction [14]. The small sized $<111>$ SiNWs were difficult to obtain in the VLS process. But in our result, it was shown that the small-sized $<111>$ SiNWs could be obtained from cluster assembly possibly.

Moreover, model (a) showed the largest binding energy among the five candidates. It was evidence that the cluster assembly in $<100>$ direction was not energy favored. So the SiNW in $<100>$ direction reported would be formed by cluster assembly less possibly. But etching was an available method to manufacture the $<100>$ SiNWs from Si substrate [15]. Binding energies of model (b), (c) and (d) are less than that of model (a) but larger than that of model (e). It means that the cluster assembly in these models led to meta-stable configurations and also could be obtained in experiments with a less distribution.

In further analysis it was noticed that Si29 cluster showed the symmetry close to Td space group. The cluster assembly in $<111>$ direction could take place in 4 equivalent orientations. Consequently we built two structures with 3 Si29 clusters, displayed as Figure 3(a) a corner-like structure and Figure 3(b) a chain-like one. When the bonding happened on neighboring laterals of the central cluster, a corner-like structure would be obtained. On the contrary, a chain-like structure would be observed when the assembly took place on both sides of the central cluster. It was worth noting that the bonding was assumed to take place on the $<111>$ facets due to above results.

The binding energy was defined as $\mathrm{E}_{\mathrm{tot}}-3 * \mathrm{E}_{\mathrm{si2}}$. Interestingly, from the comparison we found that the binding energy of corner-like structure was larger than that of the chain-like model by $0.72 \mathrm{eV}$. The formation of chain-like structure was energy favored, compared with the cornerlike one. When the chain-like assembly took place several times a CDSiNWs should be formed. However, the cluster assembly in $<111>$ direction would not result in straight wires merely. Tetrapod structure or larger congregated cluster would be obtained in the assembly, either. Consequently the assembly of clusters may be a reason of the cluster growth.

Above results were interesting as the cluster assembly

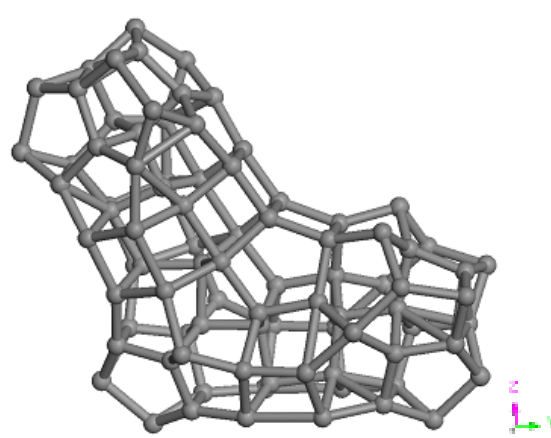

(a)

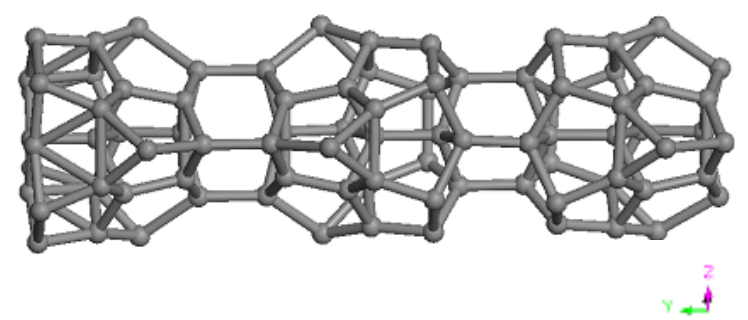

(b)

Figure 3. Assembly result of three Si29 clusters, (a) is a corner-like structure and (b) is a chain-like one.

showed the CDSiNW the possible growth process of the small-sized $<111>$ SiNW. The corner-like boding between the silicon clusters was not the most energy favored, but was the evidence of the cluster assembly in two or three dimensions.

\section{Conclusion}

As a conclusion, the assembly of the medium-sized clusters was important to the SiNW growth. The pristine clusters assemble automatically and a direction preference was revealed. The assembly of Si29 clusters would form a CDSiNW in <111> direction more possibly, and the direction preference would result in a tetrapod structure or zigzag structure besides of a CDSiNW. Moreover, the cluster growth as well as the SiNW growth was shown as a result of cluster assembly. It means that when the template or environment is suitable, the CDSiNW or some other cluster-derived materials could be obtained in experiments.

\section{Acknowledgements}

This work is supported by Natural Science Fund of Shanghai (10ZR1400200), and the author thanks the beneficial discussion with Dr. Lijuan Zhao and Prof. Huaizhong Xing.

\section{REFERENCES}

[1] Y. Cui, Z. Zhong, D. Wang, W. U. Wang and C. M. Lie- 
ber, "High Performance Silicon Nanowire Field Effect Transistors,” Nano Letters, Vol. 3, No. 2, 2003, pp. 149152. doi:10.1021/nl025875l

[2] Y. Cui and C. M. Lieber, "Functional Nanoscale Electronic Devices Assembled Using Silicon Nanowire Building Blocks,” Science, Vol. 291, No. 5505, 2001, pp. 851-853. doi:10.1126/science.291.5505.851

[3] X. H. Sun, N. B. Wong, C. P. Li, S. T. Lee and T. K. Sham, "Chainlike Silicon Nanowires: Morphology, Electronic Structure and Luminescence Studies,” Journal of Applied Physics, Vol. 96, No. 6, 2004, pp. 3447-3451. doi:10.1063/1.1782958

[4] F. Sacconi, M. P. Persson, M. povolotskyi, L. Latessa, A. Pecchia, A. Gagliardi, A. Balint, T. Fraunheim and A. D. Carlo, "Electronic and Transport Properties of Silicon Nanowires," Journal of Computational Electronics, Vol. 6, No. 1-3, 2007, pp. 329-333. doi:10.1007/s10825-006-0138-y

[5] H. Scheel, S. Reich and C. Thomsen, "Electronic Band Structure of High-Index Silicon Nanowires,” Physica Status Solid (b), Vol. 242, No. 12, 2005, pp. 1-6.

[6] X. T. Zhou, J. Q. Hu, C. P. Li, D. D. D. Ma, C. S. Lee and S. T. Lee, "Silicon Nanowires as Chemical Sensors," Chemical Physics Letters, Vol. 369, No. 1-2, 2003, pp. 220224. doi:10.1016/S0009-2614(02)02008-0

[7] D. D. D. Ma, C. S. Lee, F. C. K. Au, S. Y. Tong and S. T. Lee, "Small-Diameter Silicon Nanowire Surfaces," Science, Vol. 299, No. 5614, 2003, pp. 1874-1877. doi:10.1126/science.1080313

[8] W. Park, G. Zheng, X. Jiang, B. Tian and C. M. Lieber, "Controlled Synthesis of Millimeter-Long Silicon Nanowires with Uniform Electronic Properties," Nano Letters, Vol. 8, No. 9, 2008, pp. 3004-3009. doi:10.1021/nl802063q

[9] Y. Cui, L. J. Lauhon, M. S. Gudikesen, J. Wang and C. M. Lieber, "Diameter-Controlled Synthesis of Single-Crystal Silicon Nanowires,” Applied Physics Letters, Vol. 78, No. 15, 2001, pp. 2214-2216. doi:10.1063/1.1363692

[10] J. Yan, L. Yang and M. Y. Chou, "Size and Orientation Dependence in the Electronic Properties of Silicon Nanowires,” Physical Review B, Vol. 76, No. 11, 2007, Article ID: 115319. doi:10.1103/PhysRevB.76.115319

[11] I. Zardo, L. Yu, S. Conesa-Boj, S. Estrade, P. J. Alet, J. Rossler, M. Frimmer, P. R. Cabarrocas, F. Peiro, J. Arbiol, J. R. Moreante and A. F. Morral, "Gallium Assisted Plasma Enhanced Chemical Vapor Deposition of Silicon Nanowires,” Nanotechnology, Vol. 20, No. 15, 2009, pp.
155602-155610. doi:10.1088/0957-4484/20/15/155602

[12] S. A. Fortuna and X. Li, "Metal-Catalyzed Semiconductornanowires: A Review on the Control of Growth Directions," Semiconductor Science and Technology, Vol. 25, No. 2, 2010, pp. 024005-024020. doi:10.1088/0268-1242/25/2/024005

[13] Y. Wu, Y. Cui, L. Huynh, C. J. Barrelet, D. C. Bell and C. M. Lieber, "Controlled Growth and Structures of Molecular-Scale Silicon Nanowires," Nano Letters, Vol. 4, No. 3, 2004, pp. 433-436. doi:10.1021/nl035162i

[14] Y. Wu, H. Yan and P. Yang, "Semiconductor Nanowire Array: Potential Substrates for Photocatalysis and Photovoltaics,” Topics in Catalysis, Vol. 19, No. 2, 2002, pp. 197-201. doi:10.1023/A:1015260008046

[15] Z. Huang, T. Shimizu, S. Senz, Z. Zhang, X. Zhang, W. Lee, N. Geyer and U. Gosele, "Ordered Arrays of Vertically Aligned [110] Silicon Nanowires by Suppressing the Crystallograpgically Preferred $<100>$ Etching Directions,” Nano Letters, Vol. 9, No. 7, 2009, pp. 2519-2525. doi:10.1021/nl803558n

[16] X. L. Ma, Y. L. Zhu and Z. Zhang, "Growth Orientation of One-Dimensional Silicon Nanowires Prepared by Thermal Evaporation,” Philosophical Magazine Letters, Vol. 82, No. 8, 2002, pp. 461-468. doi:10.1080/09500830210144391

[17] H. Yorikawa, H. Uchida and S. Muramatsu, "Energy Gap of Nanoscale Si Rods," Journal of Applied Physics, Vol. 79, No. 7, 1996, pp. 3619-3621. doi:10.1063/1.361416

[18] J. D. Holmes, K. P. Johnston, R. C. Doty and B. A. Korgel, "Control of the Thickness and Orientation of Solution-Grown Silicon Nanowires,” Science, Vol. 287, No. 5457, 2000, pp. 1471-1473. doi:10.1126/science.287.5457.1471

[19] W. Ong, E. S. Tok, H. Johll and H. C. Kang, "Self-Assembly, Dynamics and Structure of Si Magic Clusters," Physical Review B, Vol. 79, No. 23, 2009, Article ID: 235439. doi:10.1103/PhysRevB.79.235439

[20] T. Sekiguchi, S. Yoshida and K. M. Itoh, "Self-Assembly of Parallel Atomic Wires and Periodic Clusters of Silicon on a Vicinal Si(111) Surface,” Physical Review Letters, Vol. 95, No. 10, 2005, Article ID: 106101. doi:10.1103/PhysRevLett.95.106101

[21] Z. Jing and J. L. Whitten, “Ab Initio Studies of Si(100) Surface Reconstruction,” Surface Science, Vol. 274, No. 1, 1992, pp. 106-112. doi:10.1016/0039-6028(92)90104-E 